\title{
Study on the Oil Painting Language Study of the Image of Lotus in Ancient Poems
}

\author{
Haiyan Huang \\ Academy of Arts \& Design, Sichuan University of Arts \& Science, Dazhou, China \\ Email: huanghaiyan200168@163.com
}

How to cite this paper: Huang, H. Y. (2020). Study on the Oil Painting Language Study of the Image of Lotus in Ancient Poems. Open Journal of Social Sciences, 8 , 111-119.

https://doi.org/10.4236/jss.2020.811011

Received: September 29, 2020

Accepted: November 10, 2020

Published: November 13, 2020

Copyright $\odot 2020$ by author(s) and Scientific Research Publishing Inc. This work is licensed under the Creative Commons Attribution International License (CC BY 4.0).

http://creativecommons.org/licenses/by/4.0/

(c) (i) Open Access

\begin{abstract}
Take the image of lotus in ancient poems as the carrier, and analyze the cultural implication of lotus imagery. Through the internal connection between lotus visual text and image language, the metaphorical image of lotus in ancient poetry and the image interpretation of lotus in oil painting language are used as the starting point to describe the artistic conception in literary works in an image way. There are four parts to study the image of lotus in ancient poems. Observing the state of things and experiencing the feelings of things; using the spirit of lotus and lotus to express the spirit of poetry, finding the aesthetic conception of poetry, co-constructing the strategy of image and text isomorphism, and fitting the personalized oil painting creation language with both pictures and text, it can help readers to build a new aesthetic perception and poetic comprehension to realize the cross-cultural resonance of poetry and painting imagery.
\end{abstract}

\section{Keywords}

Ancient Poetry, Image of Lotus, Oil Painting

\section{Interpretation of Related Concepts of "Lotus Image"}

Language is a variety of expression symbols for people to communicate. Broad language includes text language, body language, image language and all symbols of human communication. Image is an intuitive visual presentation. As a cultural symbol communication, it is one of the main language forms of human beings. It has strong "readability". Since the moment of its birth, language is closely related to the time process and spatial region. Language is inherent in the time and space of coming (Pei, 2012).

Poetry is composed of different images to form the artistic conception of the whole poem. The words are disconnected from the meaning and convey the 
poet's emotions. The poet fixes the image in his mind through words and algae, provides readers with space for association, and jointly undertakes the task of communicating thoughts and feelings. Jiang Yin understood "imagery" in "Modern Interpretation of Classical Poetics" as: "The image structure composed of one or more image images processed by the poet's feelings and consciousness and possessing a certain poetic self-sufficiency constitutes a poetic text" (Jiang, 2003). Imagery relies on the object image and is based on the object image, but it is not the true restoration of the object image. Although the object image remains the same, different images are formed due to the integration of different subjective feelings.

The image of lotus has rich image connotation, symbolic meaning and expression methods in ancient Chinese poetry: prototype image, natural image, aesthetic image, and so on.

\section{The Cultural Implication of "Lotus Image"}

The theme of lotus is favored by men of literature and writing. It carries an important archetype image in ancient Chinese poetry culture: the image of gentleman of lotus; the image of beautiful lotus; the image of love and marriage of lotus; lotus represents beautiful things and beautiful situational images; the image of sorrow, sadness and resentment of lotus; the image of nostalgia and nostalgia of lotus are presented in a dynamic trend.

\subsection{Origin of Lotus Imagery}

In the earliest book of songs in China, lotus image appeared, which means women, and men and women love. In Quyuan's $\mathrm{Chu} \mathrm{Ci}$, lotus form: one is a gentleman with high quality; the other is a symbol of melancholy and sad love. Lotus, as a "fragrant flower", symbolizes the poet's character and political destiny. Until the Wei, Jin, southern and Southern Dynasties continued to Tang Dynasty, lotus was also known as a gentleman; the Fu of Luo God by Cao Zhi, a literary scholar in the Three Kingdoms period, reflected the symbol of lotus continuing his sad and sad love. In short, "Lotus" means the symbol of gentleman and love. Like plum blossom, orchid, bamboo and chrysanthemum, it carries people's desire for love and a better life, expresses the eternal love and life, and consolidates the soul.

\subsection{Lotus Image in Tang Poems}

The image of and lotus in Tang Dynasty is rich in connotation: it symbolizes beauty and love, peace and tranquility, and the personality of gentleman. Literati used it to complete the image function of lyricism, symbolism, etc., to open up new artistic conception and imagination. The lotus-picking literature also shifted from graphic depiction to emphasizing charm, prosperity and sustenance. The image of lotus and lotus departed from the numeral meaning of female reproductive worship, and developed from the beauty of appearance to a 
symbol of affection for female love and compassion, pursuing noble aspirations and rich personality. It was formed as a character symbol of "Clivia" in Song Dynasty.

\subsection{The Lotus Image in Song Ci (a Type of Lyric Poetry in Song Dynasty)}

The image of lotus in "Complete Song Ci": "Lotus" symbolizes beauty; entrusts auspicious and beautiful ideals; borrows lotus verses to convey love, mainly lingering lovesickness, with exquisite rhyme and eternal charm. Zhou Dunyi's "Lotus" in "Love Lotus" is likened to "the gentleman of flowers". He is good at taking the noble characteristics of lotus, borrowing the natural attributes of plant lotus, and the law of growth structure as a metaphor for gentleman's personality posture. The literature of the Song Dynasty mainly used clear language arts and techniques to reveal the aesthetic connotation of the image of lotus.

The image of lotus originated in "The Book of Songs" and "Chu Ci". It became the main body of aesthetics during the Wei and Jin dynasties. The Tang Dynasty flourished, and the Song Dynasty transferred personality from the gentleman in literature to the image of withered lotus in Yuan Xiaoling. The visual language of each era corresponds with different image forms.

\section{A Probe into the Language and Image Language of "Lotus Imagery"}

\subsection{A Probe into the Context of Poems and Images}

Vision is our way of life. We are facing the era of visual culture. Cultural symbols tend to be images. Image production involves the political, technological, commercial and aesthetic themes of modern society. The "Image Age" changes people's reading habits. Modern and contemporary is an "image world era". From the perspective of iconology, interpreting text with images as objects, and studying the artistic conception in literary works is a relatively new research field. Drawing images is an intuitive way of visual presentation. Images and text are like a systematic project. It integrates image, text, and ideology, and is a concentrated presentation of physical images and inner spiritual images of things themselves, including images, spirits, and words. Image text does not exist in isolation, it is intertwined with images.

"The Book of Songs" describes the natural ecology of the "Lotus" landscape, showing the relationship between man and man, man and nature. The "Lotus" in "Chu Ci" reflects the primitive charm of totem worship, the cultural custom of sacrificing witchcraft, and the special role of vegetation witch doctors. Artists, as participants in a unique visual culture, use brushes to understand the world, understand poetry, and reveal the image and ideology of visual art to link text. It is a textual representation of creative consciousness that reflects the imaginative relationship between individuals and their actual existence environment. When simple text is difficult to effectively convey the content of the author's expres- 
sion, matching with relevant picture information will make the text content more vivid and easy to interpret. This expression of language and time and space reflects the interrelationship between language and culture, thinking and action. We will explore how language and image art turn from time to space, from depth to plane, from the whole to fragments for reasonable imitation and reference. From the cultural system of lotus and lotus poems, we can look for the visual representation of lotus and lotus imagery and the formal characteristics of verbal expression: to coordinate and resolve the contradiction between "form" and "content" between the tension of "language" and "image". The conversion and integration of "text" and "context" explores the "language image" and "graphic image" produced by poetry culture and visual art oil painting, as a research platform between literature and image.

\subsection{The Relation between the Culture of Lotus Poems and the Image Language of Oil Painting}

Our country's lotus and lotus poems have a rich cultural heritage, based on lotus culture; we should grasp the essence of Chinese poetry and poetry. Only when we have a deep understanding of the traditional culture of lotus and lotus poems, a solid foundation, and sufficient confidence, and transform the cultural connotation into our own self-cultivation, can we have depth, breadth, and thickness in our creations, and achieve the goal of taking the cultural heritage of the national lotus and lotus poems. The two have both commonality and individuality. The cultural heritage of poetry is the foundation of creation and innovation. The same is the subject of "Lotus". There are many works in China that express the image of lotus and lotus in Chinese painting, but few people use western oil painting techniques. However, no matter what method is adopted, it depends on personal emotional factors. Don't indulge in the photocopying of "graphics", understand the growth habits of lotus and lotus, observe its four seasons growth patterns, and continue to accumulate experience. The morphological expression of all objects conforms to the heart, corresponding to the meaning of the "Lotus" image, and is confident about its attitude and posture, breaking the shackles of traditional painting, establishing a new system, and presenting a new dynamic picture development situation.

\subsubsection{Poetic and Painting Metaphors of the Image of Lotus in Ancient Poems}

The art form of "Fu Bi Xing" in "The Book of Songs" is ingeniously corresponding to the lines of line drawing, fine brushwork and freehand brushwork of Chinese painting, and the sketch, realism and abstraction of oil painting. Through the poetic lotus context, the painting meaning of lotus in ancient poems and painting the poetic meaning of the image of lotus in the middle is revealed, and the homogeneity of poetry and painting is found, and the integration and difference between poetry and painting are explored.

The ancient poet Wang Wei proposed that "there are pictures in poems" and 
"there are poems in pictures". Pan Huanlong mentioned in "Woyuan Poetry", "In the past it was said that there were paintings in poems and poems in paintings. However, those who paint water cannot paint the sound of water, those who paint objects cannot paint shadows of objects, and those who paint people cannot paint people. Poetry cannot be painted, so it is more wonderful than painting things". It means that Chinese poetry and painting have the same origin: poetry is art of time, painting is art of space. The two have a common way of thinking, a common structure and a common pursuit of artistic conception. The image language is the virtual object recorded by the medium of poetry, the reproduction and creation of the poetic context, and it belongs to experience. In the process of dissemination, there are also constraints of the creators' self-cultivation, cultural background, and literary background and painting skills. Readers cannot fully interpret and present the visual image created by the language of poetry. They can only read through images and rely on their own cultural accomplishment and knowledge. The system makes a moderate or limited interpretation of the signifier of poetry and painting symbols, recreates and constructs new cognition. While creating visual effects, the creator pursues the transcendence of the picture space, breaking through the two-dimensional space of the picture, and pursues deep-level poetic connotation.

Painting, as the disseminator of poetry information, creates image symbols, readers as information receivers, the common experience of painters and viewers interprets the signifier of image language. Zhu Da, Shi Tao, Zhang Daqian and Western master Monet stand on different painting stances. There is no gap between the creator and the viewer in the expression of objects, the interpretation of culture, and the exchange of hearts due to differences in the performance of paintings. From ancient times to the present, everyone has loved lotus and used it to express feelings and constantly seek for the "Lotus" image language suitable for oneself.

\subsubsection{Analysis of Oil Painting Language of Lotus Image}

French impressionist painter Monet's "Water Lily" works, in free time and space environment, makes full use of natural external light, and depicts the internal connection between water, color and form through the points, lines and surfaces produced by brushstrokes Water lily form. The two-dimensional lotus and lotus works expand the depth of the visual space. The air is filled with lifelike lotus scenes, which have obvious oriental freehand colors, vivid and harmonious, and give people a beautiful enjoyment.

Wu Guanzhong's "Lotus" works "Red Dragonfly". He advocated "exploring nationalization in oil painting and seeking modernization in ink and wash" to alleviate the contradiction between lotus and lotus culture and language images (Yin, 1983). The picture is during the Cultural Revolution, the winter wind is bitterly cold, the remaining lotus is not harvested, some have been dumped, frozen into zombies, still standing upright. It borrowed the scene of red Dragonfly mourning the unjust souls. He uses concise, refined, lively and generalized dots, 
lines and surfaces, simple and elegant or strong folk color elements to study the connotation of national culture and painting. Finding the right entry point between tradition and modernity, focusing on and pursuing the beauty of form, combining concrete and abstract factors, creating works with spiritual connotations of lotus visual schema.

Ouyang abides by the principle of the same source of painting and calligraphy, using fiction to bring truth, sentiment to bring pen, freehand brush, to blend dryness, wetness, coldness and warmth, lightness and severity, shape and color into one, casting spirit and affection in one furnace. The oil painting "Pool" uses a poetic pen to transform the context of ink painting into the language of imagery oil painting. It combines traditional Chinese ink painting techniques with the light and color of impressionism through the techniques of "being well-informed" and "creating the environment". Oil painting images give oriental charm, creating meaningful graphical language to express the artistic conception of lotus and lotus. Monet's "Water Lily" works maintain the original appearance of the object, pursuing the charm of light and color. The two use different artistic concepts and creative methods to express the disagreement of the same object.

\subsection{The Oil Painting Language Exploration of the Image of Lotus}

From the vertical and horizontal aspects, systematically analyze and sort out the development and evolution of the painting language of ancient Chinese poetry, lotus and lotus, focus on the practice creation of lotus painting language, and find a new oil painting language suitable for personal development from the cultural context of ancient poetry.

\subsubsection{If You Can't Do It, It's Your Own Method: To Observe the State of Matter and Experience the Situation}

Images and language are both carriers that show the poetry. The presentation of lotus and lotus images meets the aesthetic needs of understanding the literary image of lotus and lotus poems. The image is the "virtual finger" and the language is the "real finger". The image serves as a tool and carrier, and the creator understands the physical condition by observing the physical state. From the pursuit of objective reproduction of the image, the realization of "lotus painting does not draw the shape", learning from the heart of the ancients and the ancient ruins, the prototype is lifelike but not full of spirit. Using the observed objects to extract the carrier images of emotional expression, creating a mood, and constructing a new image world in which the visual image and the mental image are merged.

Folk Songs in Han Dynasty "Jiangnan": "The south of the Yangtze River can pick lotus, and the lotus leaves He Tiantian. The fish plays in the lotus leaves, the fish plays in the east of the lotus leaves, the fish plays in the west of the lotus leaves, the fish plays in the south of the lotus leaves, and the fish plays in the north of the lotus leaves". Wang Changling's "The Song of Picking Lotus": "The lotus leaf skirt is cut in one color, and the hibiscus is opened to the sides of the 
face. I can't see it when I enter the pool in chaos, I only feel someone is coming after hearing the song. Wang Bo's "Pairing the Lotus" describes the aptitude of the lotus itself, its color, posture, and charm. Poets can't help but use imagination and association to describe the slim lotus and its surrounding environment. Through the reading of poems, the contained lotus, fish, enchanting lotus, and dense lotus leaves are used to express lifelike objects in the form of oil paintings, so that visual images and words resonate, convey the poetic mood, and entrust beauty and love.

\subsubsection{There Is Me without Me, and There Is Nothing to Do, to the Remnant Lotus Has No Flowers, and Its Quality Is Proud}

The lotus is the traditional "Virtue" flower in our country. The lotus that lent the silt but not stained as the life attitude and aesthetic ideal of life and death, desire, its original external aesthetic care, gradually transformed into the internal personality comparison, injecting "self” life color.

Li Qunyu’s “Lian Ye" (“Quan Tang Poems” Volume 570): “The root is jade in the mud, the heart bears the dew from the pearl. I plant it under Juntang and bury RenChunpu". It expresses the poet's noble temperament and does not follow the flow. BaiJuyi's "Sense of White Lotus" ("Quan Tang Poems" Volume Four and Five Two): Using the white lotus as a metaphor for the fate of people in disaster, praises the white lotus character, and outlines the outstanding protagonist's pure and flawless image of the white lotus. Li Bai (Ancient Style) 26 ("Quan Tang Poetry" Volume 1), borrowed the noble and sweet fragrance of lotus, symbolizing his talent experience, BaiJuyi”s "New Planting Lotus in Jingzhao Mansion" ("Quan Tang Poetry" Volume 42 Four): Outline the image of the protagonist outstanding. Marble "Xiaoliangzhou-Autumn": "Furong reflects the water, chrysanthemum yellow, full of autumn light. Heron hiding under dry lotus leaves". Depressed autumn scenery is depicted with dry lotus. Liu Bingzhong's "Dry" "Lotus Leaf": Dry lotus leaves, the color is pale, the old handle is swaying in the wind. The fragrance is reduced, and the more yellow. Because of the frost last night, the loneliness is on the autumn river. It thrives with the fiasco of the dry lotus leaves. Use simple brushwork to outline a desolate and Zen-inspired picture of the fall of the lotus on the autumn river. Li Shangyin's "Suluo's Pavilion Sending Love to Cui Yong and Cui Gong" in the Tang Dynasty: "Bamboo docks are dust-free and water is clear, and love is separated. Autumn yin does not dissipate the frost and fly late, leaving the dry lotus to listen to the rain". Tang poetry uses "withered lotus" to convey personal sadness or political feelings. Xiaoling in Yuan Dynasty elevated it to a high level of care for society and life. It fits the literati's aesthetic mentality of autumn.

Color is the external expression of individual language elements with meaningful forms. It can arouse people's visual attention and emotional resonance in an instant, and can best detect the inner emotional state of the creator. The "Dance of Lotus" series of oil paintings show the tenacious vitality of lotus and lotus from three stages: blue series, red series and blank series. The blue picture 
is relatively light, simple and peaceful, and the cool and bleak natural color system expresses the nobility and restraint of lotus. The red series is based on the unique girl's experience, social experience, and emotional changes (Terence Hawkcs Structuralism and Semiotics, 1987). Compared with the bright, elegant and enchanting of Shimizu Hibiscus, the lotus leaf, lotus stem, and lotus are intertwined with each other, and the dry brown is interwoven with red, blue, purple and other colors. Blank space is a kind of ethereal, which is a kind of artistic conception, ethereal realm with the continuation of life. The attention to the withered lotus in the late autumn season is actually the attention to the life itself. In the creation, the scene is in love, the sound, the scene, and the sentiment are combined into one, and the residual lotus is used to entrust the passing of beauty and love. It contains the souvenir of the aging of life, the sadness of the withering of beautiful things and other emotions. The decline of lotus is associated with life to express the poet's parting and missing relatives, express the sadness and imitation of homesickness. When pitying and mourning the loss of the fragrance of lotus, the crippled lotus needs to strive for greater life space and contend with nature. The power of lotus and lotus corresponds one-to-one with the extension of the creator's heart, forming a new interpretation of text and image.

\subsubsection{Intangible, Intentional, Silent and Sound: To Write the Spirit in} Form and Express the Spirit with Emotion

The "Dance of Lotus" series of works explore the situation, mood, and gestures in his paintings from the visual psychological characteristics, and work together to form the "force" movement of the work: from the contrast between reality and existence; detailed and general contrast; strong and weak Contrast between traditional culture and modern discourse. The natural growth pattern of the lotus from rooting, budding, flowering, and fruiting withering, highlights the stubborn and upright character of the lotus. The emotions of art creators come from the individual's different understanding of the state of nature: using lotus lotus as the carrier, the individual has a special affection for lotus lotus, observing and experiencing with heart, moving with "emotion" and borrowing Born to work with "emotion", build emotional bridges, transmit power, and record its state. In the form of expression, emphasis is placed on planeness, consciously neglecting space, and the traces of the creation pattern are weakened, annotating new modern language symbols-coquettish flowers, graceful and graceful torso, vigorous lotus, red and heavy lotus leaves and other painting elements are harmonious. Naturally enter the cultural context of his own painting image language, showing his individuality.

\section{Conclusion}

National culture is facing the impact and collision of world culture, and the language is turning to the trend of diversification. We study the language of oil painting creation based on the image of lotus and lotus in ancient poems, explore the artistic conception in literary works from the perspective of images, 
and build language maps together to explore the schema of each individual's spiritual image through time and space. Adopting continuous attempts, combining and borrowing feasible methods to create your own unique spiritual schema, looking for a poetic aesthetic conception, co-constructing the strategy of image and text isomorphism, and matching the personalized oil painting creation language interpretation method with both pictures and texts, help readers construct brand-new aesthetic cognition and poetic comprehension, and find spiritual support in the form of combination of text and image in terms of values, thinking styles and cultural concepts, so as to achieve cross-cultural resonance between poetry and painting imagery. This is the discussion of the creation language of lotus painting in China. At the same time, it also provides research ideas for us to explore and develop the oil painting creation language on related themes.

\section{Fund Project}

The key project of Sichuan Provincial Department of Education "The Study of the Language of Oil Painting Creation of "Lotus" in Ancient Poems" (18SA0219).

\section{Conflicts of Interest}

The author declares no conflicts of interest regarding the publication of this paper.

\section{References}

Hawkcs, T. (1987). Structuralism and Semiotics (pp. 23-24). Qu Tiepeng Translated, Shanghai: Shanghai Translation Publishing House Press.

Jiang, Y. (2003). Modern Interpretation of Classical Poetics (pp. 27). ZHBC.

Pei, W. (2012). Ingenious Words on Time and Space (pp. 278-279). Beijing: Qiaowu Press.

Yin, H. Y. (1983). Memoirs of Repin. Beijing: People's Fine Arts Publishing House Press. 